\title{
An Efficient and Enjoyable Way for Physical Fitness Development among Women at Midlife
}

\author{
Omri Ezra' ${ }^{1}$, Sharon Tsuk ${ }^{1}$, Alon Eliakim ${ }^{1,2}$, Yoav Meckel ${ }^{1 *}$ \\ ${ }^{1}$ The Academic College at Wingate, Wingate Institute, Netanya, Israel \\ ${ }^{2}$ Child Health and Sport Center, Pediatric Department, Meir Medical Center, Sackler School of Medicine, Tel Aviv University, Tel \\ Aviv, Israel \\ Email: ^meckel@wincol.ac.il
}

How to cite this paper: Ezra, O., Tsuk, S. Eliakim, A. and Meckel, Y. (2020) An Efficient and Enjoyable Way for Physical Fitness Development among Women at Midlife. Health, 12, 943-953.

https://doi.org/10.4236/health.2020.12807

Received: July 10, 2020

Accepted: August 10, 2020

Published: August 13, 2020

Copyright (c) 2020 by author(s) and Scientific Research Publishing Inc. This work is licensed under the Creative Commons Attribution International License (CC BY 4.0).

http://creativecommons.org/licenses/by/4.0/

\begin{abstract}
The aim of the study was to examine the effect of Newcomb ball (also known by the name of catchball) training on the physical fitness of women at midlife. One hundred and eighteen women, aged 29 - 45 yrs, were assigned to an experimental group (EG) or a control group (CG), and completed two similar sets of tests before and after seven months of training. The EG performed two Newcomb ball training sessions per week that included technical and tactical drills with and without the ball, small sided games and full games at different variations. The CG remained non-active throughout the seven months. Leg power, arm power, agility, speed, and aerobic scores were significantly higher for the EG compared to the CG at baseline ( $p<0.001$ for all). EG showed significant training-related improvement in arm power $(5.3 \pm 0.9$ vs $5.6 \pm 0.9$ $\mathrm{m})$, agility ( $10.68 \pm 0.95 \mathrm{vs} 10.37 \pm 0.89 \mathrm{sec})$, speed ( $4.11 \pm 0.45$ vs $4.01 \pm 0.33$ sec), and aerobic fitness $(563 \pm 328$ vs $680 \pm 378 \mathrm{~m})(\mathrm{ES}=0.45-0.61, p<$ 0.001 for all), while no significant changes, except for a significant increase in arm power $(E S=0.42, p<0.05$ ), were found for the CG in any of the other fitness variables. The findings indicate that amateur Newcomb ball training can be used as an efficient and enjoyable tool for fitness development among midlife women.
\end{abstract}

\section{Keywords}

Team Sport, Training, Strength, Aerobic Fitness

\section{Introduction}

Newcomb ball (also known as Catchball in some countries) is a team ball game played mostly by women, and is similar in its basic characteristics and rules to 
the game of volleyball [1] [2]. Although in both games, the player's objective is to deliver the ball over the net to the opposing team's court, in Newcomb ball the players are allowed to catch and hold the ball for one second, whereas in volleyball the players are only allowed to hit the ball. The court and net sizes, point accumulation rules, number of players, and positions are identical in both games.

Newcomb ball was first introduced in 1895 [3], but has never been officially categorized as a competitive sport with a structured active league. In Israel, however, during the last two decades a growing number of women at midlife have joined together and established teams that meet on a weekly basis to practice Newcomb ball. Hence, it has formally become a registered sport that is now played in an organized league setting. At present, the participants in the Israeli Newcomb ball league are woman in the age range of 30 to 50 years, who in most cases practice twice a week. These players are amateurs at best and are a long way from the physical status and performance capabilities of typical trained female volleyball players [4] [5]. The growing activity of this sport among women in this age range may be encouraging since the common fitness level is usually low to moderate while the level of coronary heart disease risk factors is increasing in this population [6]. This is especially encouraging since previous studies showed higher motivation for activity among females of team compared to individual sports [7]. In line with this, it was shown that several weeks of team sport such as soccer practice in untrained women significantly improved aerobic fitness and other cardiovascular variables, more than in a comparable running group and a non-active control group (age 20 - 47) [8]. It should also be emphasized that, in contrast to other team sports like soccer or basketball, no body contact occurs between players in Newcomb ball. Obviously, this may reduce possible injury risk and may offer a safer team sport activity for older untrained population.

Previous studies have provided comprehensive data regarding the physical profile of young female volleyball players [9] [10]. Some of these studies showed that female volleyball players significantly improve various fitness variables following the use of relevant training methods [5] [11] [12]. However, to the best of our knowledge, not a single study had investigated the physical capabilities of Newcomb ball players or examined the effect of training on their physical fitness level.

Therefore, the aim of the present study was to determine the effect of Newcomb ball training on the physical fitness of women at midlife in Israel. We hypothesized that two sessions per week of Newcomb ball training will induce improvement in different physical fitness components among these women.

\section{Material and Methods}

\subsection{Participants}

A total of 118 women (age range $29-45 \mathrm{yrs}$ ) volunteered to participate in the 
study. The participants were assigned to one of two different groups: 1) Experimental group (EG) - 82 amateur Newcomb ball players, and 2) Control group (CG) - 36 non-active women. All the participants were healthy and did not use medications or supplements of any kind. The EG included members of nine different teams (of the 12 league teams) from the women's Newcomb ball league in the center of Israel. Most players had training experience of three to five years with their teams. These players practiced twice a week, or practiced one time and played one time a week, during the Newcomb ball season. If any player missed more than $20 \%$ of total practiced time, for any reason, she was dropped from the analysis and was not part of the study. The CG included women who were not involved in any individualized or organized physical activity, nor they were involved in any organized sport activity in the past. All the participants were informed of the study requirements, benefits, and risks before giving their written informed consent. The study was approved by the institutional research ethics committee.

\subsection{Procedures}

Figure 1 illustrates the time frame and the different stages of the study. During the first two weeks of September, before the beginning of the Newcomb ball training, the two groups-EG and CG-performed a pre-training series of physical and fitness testing procedures. During the summer months (June to $\mathrm{Au}$ gust), prior to the pre-training testing procedures, both the EG and the CG participants did not perform any type of organized physical activity. Upon the completion of the pre-training testing for both groups, the EG started a period of seven months of twice-a-week training and playing Newcomb ball. Each training session lasted 75 to $90 \mathrm{~min}$. Most sessions were conducted as follow: a warm-up of 15 to 20 min that included slow running, stretching exercises, basic coordination drills such as skipping, vertical and horizontal jumping and hopping without the ball. Then after, about 25 to 30 min of technical and tactical drills with the ball were performed. Lastly, the players performed 25 to $35 \mathrm{~min}$ of small sided games (usually of $3 \times 3$ ), and/or full practice games. At least one of the investigators visited one of the practice sessions once every two weeks. The EG participants did not perform any other sports activity. During this period, the CG did not perform any physical activity other than their regular daily activities
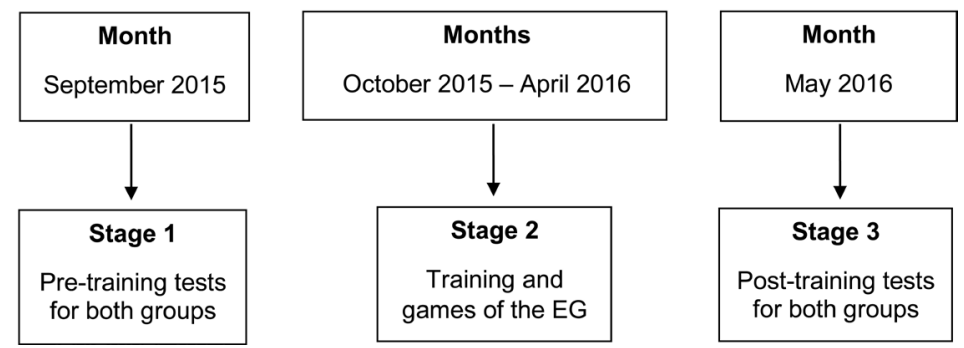

$\mathrm{EG}=$ Experimental group.

Figure 1. Scheme of the time frame and the different stages of the study. 
at work and at home. After seven months of training both groups performed a second set of post-training fitness testing, identical to the pre-training testing procedure. Overall, the study lasted nine months.

\subsection{Testing Protocol}

Pre- and post-testing was conducted on two non-consecutive days using the same protocol and under similar environmental conditions as in the training sessions. Two trials were given for each test, with two min of recovery in-between, and the better result was recorded for each test. A period of 15 min separated the different tests on each day. Running times were recorded using a photoelectric cell timing system (Alge-Timing Electronic, Austria) with an accuracy of $0.001 \mathrm{~s}$, linked to a digital chronoscope. Given that the participants in both groups were not familiar with the tests' protocols and did not perform them before, a special practice session (in some cases more than one session was required), of the different testing procedures, was performed before the pre-training testing.

On the first day of each testing set, participants performed the following three tests:

Vertical jump-Leg power test: Jump height was measured using a vertical countermovement jump (CMJ) technique with double-arm swing. The flight time, measured by an Optojump (Optojump, Microgate, Bolzano, Italy), was used to calculate the change in the height of the body's center of gravity.

Sit and reach-Flexibility test: Participants sat with legs extended on the floor and feet placed against a box with a yardstick attached to it. The participants extended their arms forward and the distance reached on the yardstick was recorded.

$20 \mathrm{~m}$ sprint run-Speed test: A $20 \mathrm{~m}$ sprint run was used to evaluate the participants' speed and acceleration ability. The participants self-started after assuming a standing ready position. Each participant performed the test individually.

On the second day, the participants performed the following three tests:

T-test-Agility test: Four cones were placed $5 \mathrm{~m}$ apart in a T shape [13]. Participants ran forward $5 \mathrm{~m}$ from the start line to the first cone, shuffled $5 \mathrm{~m}$ left to the second cone, then shuffled $10 \mathrm{~m}$ to the right to the third cone, and then shuffled $5 \mathrm{~m}$ back left to the middle cone, before finally running back to the start line.

Overhead ball throws-Arm power test: Participants held a $2 \mathrm{~kg}$ medicine ball in both hands behind the head with one leg in front. They were then asked to throw the ball using arm movement only, without the use of a leg push [13].

The $20 \mathrm{~m}$ shuttle run-Aerobic power test: The test consisted of shuttle runs at increasing speeds between two markers placed $20 \mathrm{~m}$ apart. A start speed of 8.5 $\mathrm{km} /$ hour was maintained for one min, and thereafter the speed was increased every minute by $0.5 \mathrm{~km} /$ hour before the participant withdrew from the test [14]. Aerobic fitness was calculated as the number of laps or the total distance achieved during the test.

A high test-retest reliability $(0.80-0.95)$ for most of the different tests used in 
this study was described previously [13]. Pre- and post-training evaluation also included body weight, height, resting heart rate (RHR), and systolic blood pressure (SBP). HR was also recorded during training, using a telemetry system (T31, Polar, Electro, Finland). Standard calibrated scales and stadiometers were used to determine body weight, height, and body mass index (BMI).

\subsection{Statistical Analysis}

The mean difference among the participants in the two experimental conditions at the pre-intervention stage was calculated by an independent t-test. Repeated measures ANOVA ( 2 groups $\times 2$ test dates) analysis was used to follow changes over time in fitness and physiological measurements. Post-hoc test was performed using Bonferroni adjustment for each measurement separately. Cohen's d-effect size (ES) was also performed to demonstrate the magnitude of the training effect in each group. Cohen's $\mathrm{d}<0.30$ was considered a low effect, 0.30 0.70 a moderate effect, and $>0.70$ a strong effect. Data are presented as mean $\pm \mathrm{s}$. Significant level was set at $p \leq 0.05$.

\section{Results}

Resting heart rate ( $68 \pm 8.3$ vs $73 \pm 7.1 \mathrm{~b} / \mathrm{m})$ and resting systolic blood pressure (RSBP) (113 \pm 9.6 vs $119 \pm 14.6 \mathrm{~mm} \mathrm{Hg})$ were significantly lower in the EG compared to the CG at baseline $(p<0.05)$, respectively. In contrast, no baseline differences were found in body weight or BMI between the groups (Table 1). Post-Hoc teats for fitness components showed that leg power (CMJ) (29.0 \pm 5.5 vs $21.3 \pm 4.3 \mathrm{~cm}$ ), arm power (BT) $(5.3 \pm 0.9$ vs $4.2 \pm 0.7 \mathrm{~m})$, agility (TT) (10.68 \pm 0.95 vs $12.94 \pm 2.0 \mathrm{sec})$, speed ( $20 \mathrm{~m}$ sprint $)(4.11 \pm 0.45$ vs $4.45 \pm 0.48 \mathrm{sec})$, and aerobic scores $(20 \mathrm{~m} \mathrm{ST})(563 \pm 328$ vs $337 \pm 171 \mathrm{~m})$ were significantly higher for the EG compared to the CG at baseline ( $p<0.001$ for all), respectively (Table 2).

No significant changes were found following training for either group in resting physical or physiological values, with the exception of a significant increase in body weight for the CG $(p<.05)$. EG showed significant improvement in BT $(5.3 \pm 0.9$ vs $5.6 \pm 0.9 \mathrm{~m})$, TT ( $10.68 \pm 0.95$ vs $10.37 \pm 0.89 \mathrm{sec}), 20 \mathrm{~m}$ sprint $(4.11$ $\pm 0.45 \mathrm{vs} 4.01 \pm 0.33 \mathrm{sec})$, and $20 \mathrm{~m} \mathrm{ST}(563 \pm 328$ vs $680 \pm 378 \mathrm{~m})(p<0.001$ for

Table 1. Physiological resting values of the two groups following training (mean \pm STD).

\begin{tabular}{|c|c|c|c|c|c|c|c|}
\hline \multirow{2}{*}{ Variable } & \multicolumn{3}{|c|}{ Experimental group } & \multicolumn{3}{|c|}{ Control group } & \multirow{2}{*}{$\begin{array}{l}\text { Interaction } \\
\qquad \mathrm{F}_{(1,83)}\end{array}$} \\
\hline & Pre & Post & ES & Pre & Post & ES & \\
\hline Weight (kg) & $65.5 \pm 9.6$ & $66.4 \pm 8.9$ & 0.25 & $64.5 \pm 9.4$ & $65.2 \pm 10.0^{*}$ & 0.43 & 0.28 \\
\hline BMI $\left(\mathrm{kg} / \mathrm{m}^{2}\right)$ & $23.4 \pm 3.0$ & $23.8 \pm 3.0$ & 0.03 & $24.0 \pm 3.1$ & $24.3 \pm 3.2$ & 0.11 & 0.02 \\
\hline RHR (b/m) & $68 \pm 8.3$ & $70 \pm 10.6$ & 0.31 & $73 \pm 7.1$ & $75 \pm 10.2$ & 0.25 & 0 \\
\hline RSBP (mm Hg) & $113 \pm 9.6$ & $116 \pm 10.9$ & 0.38 & $119 \pm 14.6$ & $117 \pm 15.2$ & 0.46 & $15.19^{*}$ \\
\hline
\end{tabular}

Notes: $\mathrm{ES}=$ effect size; $\mathrm{BMI}=$ body mass index; RHR = resting heart rate; RSBP = resting systolic blood pressure. ${ }^{\star} p<0.05$ for within group changes. 
Table 2. Fitness changes in the two groups following training (mean \pm STD).

\begin{tabular}{ccccccc}
\hline \multirow{2}{*}{ Variable } & \multicolumn{2}{c}{ Experimental group } & \multicolumn{4}{c}{ Control group } \\
\cline { 2 - 6 } & Pre & Post & ES & Pre & Post & ES \\
\hline CMJ (cm) & $29.0 \pm 5.5$ & $29.8 \pm 6.1$ & 0.25 & $21.9 \pm 4.9$ & $21.3 \pm 4.3$ & 0.17 \\
BT (m) & $5.3 \pm 0.9$ & $5.6 \pm 0.9^{* *}$ & 0.54 & $4.2 \pm 0.7$ & $4.4 \pm 0.7^{*}$ & 0.42 \\
TT (sec) & $10.68 \pm 0.95$ & $10.37 \pm 0.89^{* *}$ & 0.45 & $12.94 \pm 2.0$ & $12.76 \pm 1.49$ & 0.16 \\
SR (cm) & $48.8 \pm 8.9$ & $49.6 \pm 9.4$ & 0.21 & $47.2 \pm 10.2$ & $45.8 \pm 7.7$ & 0.23 \\
20 m sprint (sec) & $4.11 \pm 0.45$ & $4.01 \pm 0.33^{* *}$ & 0.45 & $4.45 \pm 0.48$ & $4.51 \pm 0.49$ & 0.26 \\
20 m ST (m) & $563 \pm 328$ & $680 \pm 378^{* *}$ & 0.61 & $344 \pm 167$ & $337 \pm 171$ & 0.06 \\
\hline
\end{tabular}

Note: $\mathrm{ES}=$ effect size; $\mathrm{CMJ}=$ counter movement jump; $\mathrm{BT}=$ ball throw; $\mathrm{TT}=\mathrm{t}$-test; $\mathrm{SR}=$ sit and reach; 20 $\mathrm{m} \mathrm{ST}=20 \mathrm{~m}$ shuttle run test. ${ }^{\star} p<0.05$ for within-group changes; ${ }^{* *} p<0.001$ for within-group changes.

all), respectively, following training (Table 2). An interaction $\left(F_{(1,78)}=10.29, p=\right.$ $0.002)$ with significant main effect was found for the $20 \mathrm{~m} \mathrm{ST}\left(F_{(1,78)}=8.13, p=\right.$ 0.006 for time, and $\mathrm{F}_{(1,78)}=17.45, p<0.001$ for group). An interaction $\left(F_{(1,78)}=\right.$ $4.11, p=0.046)$ with no main affect was found for the SR. A group main effect $\left(F_{(1,80)}=24.19, p<0.001\right)$ and interaction $\left(F_{(1,80)}=9.25, p=0.003\right)$ was found for the $20 \mathrm{~m}$ sprint results. No significant changes were found for the CG in any of the fitness variables between pre- and post-training testing, with the exception of a significant decrease in $\mathrm{BT}(p<0.05)$. Average HR during training for the EG was $134 \pm 19 \mathrm{~b} / \mathrm{m}$ (range: 109-175).

\section{Discussion}

The main finding of the present study was that seven months of Newcomb ball training induced significant improvement in a variety of fitness variables-aerobic, anaerobic, and motoric-among female amateur players in the age range of 29 45. Given that no previous study had examined the effect of Newcomb ball training, and considering the relatively low physical fitness of typical women at this age range [15], the present findings are encouraging and may suggest an approachable and convenient way to improve fitness among this unique population. Moreover, the higher baseline fitness scores of the EG over the CG in the present study confirmed that EG participants were more physically fit, even after several weeks without training during the summer period. This may demonstrate a long-term effect of Newcomb ball training, particularly when considering that most of the participants had played for several years. This may also be supported by the significantly lower health-related physiological variables-RHR and RSBP - that were found in the EG compared to the CG. However, it is possible that only the physiologically superior and naturally more talented women join Newcomb ball training, whereas women with low fitness level avoid participation in this sport. A superior fitness level was also found for trained volleyball players after several weeks of a passive off-season period compared to non-athletes of the same age [2]. 
The significant improvement in physical fitness in the EG that was found following training may demonstrate the beneficial effect of Newcomb ball training on amateur women players at midlife. Moreover, the effectiveness of training was also evidenced in the present study by the diverse effect on a wide spectrum of fitness components-aerobic, anaerobic, and motoric (Table 2). While no changes were seen in any of the CG participants, significant improvements were found in arm power, agility, speed, and aerobic fitness in the EG. The diversity in fitness improvements among the EG probably reflects the nature of the Newcomb ball game. Specifically, for successful performance, Newcomb ball players are required to have arm strength in order to throw the ball powerfully over the net to the opposing team zone. Speed and agility are also required for moving around quickly and for catching the ball before it hits the ground. Endurance may be beneficial for continuous activity and for recovering from previous sudden effort bursts mainly towards the end of the game. Indeed, as previously described, the training program of the EG in the present study followed these requirements. Although no other scientific data is available on Newcomb ball training, the significant and diverse effects of training on female players has been documented previously in other team sports. For example, trained volleyball players demonstrated a significantly superior fitness level in variables such as leg power, upper body muscle endurance, flexibility, and aerobic fitness, over non-active participants of the same age [2]. Similar varied training effects were also found in different fitness variables for amateur female soccer players following 16 weeks of twice-a-week training, compared to baseline scores [16]. The heterogeneous effect of team sport training becomes noticeable especially when placed against the single effect of conventional physical activities that focus on only one specific fitness component. For example, jogging or a systematic long-distance running training program is likely to improve the cardiovascular system and aerobic fitness of the trainees, but not anaerobic or specific motoric capabilities. In contrast, resistance training programs or systematic weight lifting usually strengthen the activated muscle groups, but do not improve aerobic fitness [17]. It should be noted that the numerous and diverse effects of Newcomb ball training on these women may result from the fact that the baseline fitness level in this unique population is usually low or moderate at best [6]. Therefore, as demonstrated in the present study, even as few as two training sessions per week were sufficient to induce significant physiological and performance changes. Surprisingly, no significant changes in leg power and flexibility were found among the EG following training. However, baseline leg power (CMJ test results) was significantly greater in the EG compared to the CG. It is possible that the baseline differences reflect chronic training effects that remained superior among the EG during the summer break. Power and jumping capabilities were previously shown to be effectively maintained during the entire season in trained women volleyball players, although resistance training was performed only during eight weeks of the pre-season period [10]. It may also be argued that 
the legs, more than other body parts, remain relatively active during the off-season period in order to perform regular daily activities, therefore maintaining their power fairly well. It is possible, however, that only women with relatively strong legs tend to join and maintain Newcomb training, and more intense training is required for further improvement.

Although the positive changes in fitness variables such as power, speed, and agility are certainly of value, the significant improvement in aerobic fitness in the present study may be considered the most encouraging finding for women at midlife. Improvement in aerobic fitness may be particularly important, since this fitness variable is highly related to general health status (particularly to the cardiovascular system) [18] [19] [20], and not just to the trainee's athletic capabilities. Previous studies have reported continuous sub-maximal training as an effective type of training for enhancement of the cardiovascular system and aerobic fitness among middle-aged participants [21] [22] [23]. However, the monotonous nature of continuous sub-maximal activity may discourage trainees from adhering to this type of training for prolonged time. In line with this, a higher motivation and enthusiasm following team compared to individual sports training was reported among adolescent females [7]. Significant improvements in aerobic fitness and cardiovascular-related parameters were previously found following team-sport training. A later study [8] demonstrated that 16 weeks of two recreational football practices per week in untrained women significantly improved aerobic fitness and other cardiovascular variables, more than in a comparable running group and a non-active control group (age 20 - 47). Therefore, together with fact that in contrast to other team sport games no body contact occurs between players in Newcomb ball, the present study's findings may offer this game as an effective and perhaps safer and more enjoyable alternative for cardiovascular and aerobic improvement among women at midlife.

An obvious limitation of the present study was that, given the large number of participant and teams, we could not follow and directly supervised individuals' daily behavior throughout the seven months of training. Consequently, we only rely on the EG players report of not doing any physical activity other than the Newcomb ball training, and the CG report of not doing any physical activity other than daily activities at work and at home. In addition, no specific instructions and recommendations were given to the participants about their nutrition habits and each of them was free to eat as she wanted. The study could also benefit from intermediate fitness measurements displaying midpoint changes (after 3 - 4 months), and in particularly from repeated fitness measurements after the following summer vacation to quantify possible detraining effects. Unfortunately, such procedure was not possible to execute due to logistical difficulties, mostly teams and players' unavailability.

\section{Conclusions}

The present study demonstrated that several months of twice-a-week Newcomb 
ball training induced a significant improvement in physical fitness among amateur female players at midlife. These changes were observed in aerobic, anaerobic, and motoric fitness variables, emphasizing the efficiency of the training and its diverse effects. The significant positive influence of Newcomb ball training on aerobic fitness is of particular importance, considering its association with general health status.

Given that conventional methods for aerobic fitness development, which usually involve continuous sub-maximal activity, may be boring to some people, Newcomb ball may represent a fun, relatively easy-to-execute and safe way to develop and maintain physical fitness among women at midlife. Nevertheless, in order to prevent possible injuries, it is recommended that training will be carried out carefully and in a gradual manner that will be suitable for such untrained population. In addition, it is also possible that for women with very low baseline fitness level, even this relatively mild intensity type of sport, is not applicable and other modalities should be used to encourage these women to increase their physical activity. Future studies may also evaluate the psychological, emotional, and sociological effects of Newcomb ball training among women at midlife.

\section{Acknowledgements}

The authors wish to thank the players and the coaching staff of each of the teams for their cooperation in this study. Special appreciation should be addressed to the participants of the CG for their efforts in performing the physical tests needed for the study.

\section{Conflicts of Interest}

The authors declare no conflicts of interest regarding the publication of this paper.

\section{References}

[1] Reeser, J.C. and Bahr, R. (2008) Handbook of Sports Medicine and Science: Volleyball. Willey-Blackwell, Hoboken.

[2] Taware, G.B., Bhutkar, M.V. and Surdi, A.D. (2013) A Profile of Fitness Parameters and Performance of Volleyball Players. Journal of Krishna Institute of Medical Science University, 2, 48-59.

[3] Paul, J.A. (1996) Lost Sport: Clara Gregory Baer and Newcomb Ball. Journal of Sport History, 23, 165-174.

[4] Fleck, S.J., Case, S., Puhl, J. and Van Handle, P. (1985) Physical and Physiological Characteristics of Elite Women Volleyball Players. Canadian Journal of Applied Sport Science, 10, 122-126.

[5] Marques, M.C., Van Den Tillaar, R., Vescovi, J.D. and González-Badillo, J.J. (2008) Changes in Strength and Power Performance in Elite Senior Female Professional Volleyball Players during the In-Season: A Case Study. Journal of Strength and Conditioning Research, 22, 1147-1155. https://doi.org/10.1519/JSC.0b013e31816a42d0

[6] Lee, S.H., Seo, B.D. and Chung, S.M. (2013) The Effect of Walking Exercise on 
Physical Fitness and Serum Lipids in Obese Middle-Aged Women: Pilot Study. The Journal of Physical Therapy Science, 25, 1533-1536.

[7] Tauer, J.M. and Harackiewicz, J.M. (2004) The Effects of Cooperation and Competition on Intrinsic Motivation and Performance. Journal of Personality and Social Psychology, 86, 849-861. https://doi.org/10.1037/0022-3514.86.6.849

[8] Bangsbo, J., Nielsen, J.J., Mohr, M., Randers, M.B., Krustrup, B.R., Brito, J. and Krustrup, P. (2010) Performance Enhancements and Muscular Adaptations of a 16-Week Recreational Football Intervention for Untrained Women. Scandinavian Journal of Medicine and Science in Sports, 20, 24-30. https://doi.org/10.1111/j.1600-0838.2009.01050.x

[9] Cahill, S. and Jones, M.T. (2010) Measurement of Body Composition and Athletic Performance during NCAA-Division I Women's Volleyball and Softball Seasons. Journal of Strength and Conditioning Research, 24, 1. https://doi.org/10.1097/01.JSC.0000367077.30318.ac

[10] González-Ravé, J.M., Arija, A. and Clemente-Suarez, V. (2011) Seasonal Changes in Jump Performance and Body Composition in Women Volleyball Players. Journal of Strength and Conditioning Research, 25, 1492-1501. https://doi.org/10.1519/JSC.0b013e3181da77f6

[11] Hedrick, A. (2007) Training for High Level Performance in Women's Collegiate Volleyball: Part I Training Requirements. Journal of Strength and Conditioning Research, 29, 50-53.

[12] Pereira, A., Costa, A.M., Santos, P., Figueiredo, T. and João, P.V. (2015) Training Strategy of Explosive Strength in Young Female Volleyball Players. Medicina, 51, 126-131. https://doi.org/10.1016/j.medici.2015.03.004

[13] Gabbett, T.J. (2008) Do Skill-Based Conditioning Games Offer a Specific Training Stimulus for Junior Elite Volleyball Players? Journal of Strength and Conditioning Research, 22, 509-517. https://doi.org/10.1519/JSC.0b013e3181634550

[14] St Clair Gibson, A., Broomhead, S., Lambert, M.I. and Hawley, J.A. (1998) Prediction of Maximal Oxygen Uptake from a 20-M Shuttle Run as Measured Directly in Runners and Squash Players. Journal of Sports Science, 16, 331-335. https://doi.org/10.1080/02640419808559361

[15] Binder, E.F., Birge, S.J. and Kohrt, W.M. (1996) Effects of Endurance Exercise and Hormone Replacement Therapy on Serum Lipids in Older Women. Journal of American Geriatric Society, 44, 231-236. https://doi.org/10.1111/j.1532-5415.1996.tb00907.x

[16] Jackman, S.R., Scott, S., Randers, M.B., Orntoft, C., Blackwell, J., Zar, A. and Krustrup, P. (2013) Musculoskeletal Health Profile for Elite Female Footballers versus Untrained Young Women before and after 16 Weeks of Football Training. Journal of Sports Science, 31, 1468-1474. https://doi.org/10.1080/02640414.2013.796066

[17] Kenney, W., Wilmore, J. and Costill, D. (2012) Physiology of Sport and Exercise. 5th Edition, Human Kinetics, Champaign, IL.

[18] Chodzko-Zajko, W.J., Proctor, D.N., Fiatarone Singh, M.A., Minson, C.T., Nigg, C.R., Salem, G.J. and Skinner, J.S. (2009) Exercise and Physical Activity for Older Adults. Medicine and Science in Sport and Exercise, 41, 1510-1530. https://doi.org/10.1249/MSS.0b013e3181a0c95c

[19] O’Donovan, G., Owen, A., Bird, S.R., Kearney, E.M., Nevill, A.M., Jones, D.W. and Woolf-May, K. (2005) Changes in Cardiorespiratory Fitness and Coronary Heart Disease Risk Factors Following 24 Wk of Moderate- or High-Intensity Exercise of 
Equal Energy Cost. Journal of Applied Physiology, 98, 1619-1625.

https://doi.org/10.1152/japplphysiol.01310.2004

[20] Oja, P., Titze, S., Bauman, A., de Geus, B., Krenn, P., Reger-Nash, B. and Kohlberger, T. (2011) Health Benefits of Cycling: A Systematic Review. Scandinavian Journal of Medicine in Science and Sports, 21, 496-509.

https://doi.org/10.1111/j.1600-0838.2011.01299.x

[21] Jones, A.M. and Carter, H. (2000) The Effect of Endurance Training on Parameters of Aerobic Fitness. Sports Medicine, 29, 373-386. https://doi.org/10.2165/00007256-200029060-00001

[22] Milanović, Z., Sporiš, G. and Weston, M. (2015) Effectiveness of High-Intensity Interval Training (HIT) and Continuous Endurance Training for $\mathrm{VO}_{2 \max }$ Improvements: A Systematic Review and Meta-Analysis of Controlled Trials. Sports Medicine, 45, 1469-1481. https://doi.org/10.1007/s40279-015-0365-0

[23] Seals, D.R., Hagberg, J.M., Hurley, B.F., Ehsani, A.A. and Holloszy, J.O. (1984) Endurance Training in Older Men and Women. I. Cardiovascular Responses to Exercise. Journal of Applied Physiolology Respiretory, Environmental and Exercise Physiolology, 57, 1024-1029. https://doi.org/10.1152/jappl.1984.57.4.1024 